\title{
Neumotórax espontáneo y secundario a barotrauma en COVID 19, serie de casos y revisión de la literatura.
}

Spontaneous pneumothorax secondary to barotrauma in COVID 19, case series and literature review.

María José Pinos Cedeño. ${ }^{1}$, Gloria Estefanía Aguiar Flores. ${ }^{2}$, María Eugenia Layedra Ajila. ${ }^{3}$ \& Galo Iván Adriano Pérez. ${ }^{4}$.

\section{Abstract}

Introduction. It is widely known that Coronavirus Disease 2019 (COVID 19) can cause a Severe Acute Respiratory Syndrome, however there are certain extrapulmonary complications that can occur in the context of these patients such as pneumothorax, which despite having a reduced incidence, some case studies have reported high mortality. Pneumothorax in patients with COVID 19 can present spontaneously or induced

\section{Resumen}

Introducción. Es ampliamente conocido que la Enfermedad por Coronavirus 2019 (COVID 19) puede provocar un Síndrome Respiratorio Agudo Severo, sin embargo hay ciertas complicaciones extrapulmonares que pueden presentarse en el contexto de estos pacientes como el neumotórax, que a pesar de tener una incidencia reducida, algunos estudios de casos han reportado una mortalidad elevada. El neumotórax en los pacientes

\footnotetext{
1 Médico Especialista en Medicina Interna y General por la Universidad Central del Ecuador (Quito/Pichincha). Médico Internista del Hospital Docente Ambato. Médico Docente de la Universidad Técnica Ambato. Correo: mj.pinos@uta.edu.ec. (D) ORCID ID: 0000-0002-1146-6784.

2 Médico General por la Universidad Autónoma de los Andes (Ambato/Tungurahua). Residente del Hospital Docente Ambato. Correo:aguiarfloresgloriaestefania@gmail.com. (D) ORCID ID: 0000-00020679-1734

${ }^{3}$ Médico General por la Universidad Nacional de Chimborazo (Riobamba/Chimborazo). Residente del Hospital Docente Ambato. Correo: eugenialayedra@ hotmail.com. (1) ORCID ID: 0000-0002-7377-0546 ${ }^{4}$ Médico General por la Universidad Nacional de Chimborazo (Riobamba/Chimborazo). Residente del Hospital Docente Ambato. Correo: gap_1medicine@ hotmail.com. (1) ORCID ID: 0000-0002-1588-6073
} 
by barotrauma by invasive and noninvasive mechanical ventilation. Objective. To determine the clinical, laboratory and imaging characteristics of a group of patients who were hospitalized for pneumonia due to COVID 19 and who in turn presented as a complication of pneumothorax, within the period February to July 2021 at the Ambato Regional Hospital. Methodology. We check the clinical reports of the patients with COVID 19 admitted to the Ambato Regional Hospital for the period of time established previously, who had a diagnosis of pneumothorax, those with a traumatic origin were excluded, obtaining 3 cases; in addition to conducting a current review of the bibliography in relation to the subject. Results. A total of 6 patients had a diagnosis of pneumothorax plus COVID 19, of which two patients were excluded because the cause of their pneumothorax was considered traumatic (traffic accidents), 1 of the patients was discarded due to insufficient data on the outcome its clinical evolution; of the rest of the three presented in the article: 2 presented spontaneous pneumothorax in a late stage of the disease, one of whom died; 1 showed a pneumothorax associated with barotrauma due to invasive mechanical ventilation with a favorable clinical course. Conclusion. Pneumothorax, despite being an infrequent complication in the spectrum of COVID-19 disease, must be addressed within the differential diagnosis when a patient presents sudden respiratory decompensation; further studies are required to determine whether pneumothorax in these patients leads to higher mortality, since the reports in the con COVID 19 puede presentarse de manera espontánea o inducido por barotrauma por ventilación mecánica invasiva y no invasiva. Objetivo. Determinar las características clínicas, de laboratorio e imagen, de un grupo de pacientes que se hospitalizaron por neumonía por COVID 19 y que a su vez presentaron como complicación neumotórax, dentro del periodo febrero a julio del 2021 en el Hospital Regional Ambato. Metodología. Verificamos los informes clínicos de los pacientes con COVID 19 internados en el Hospital Regional Ambato del periodo de tiempo establecido con anterioridad, que tuvieran diagnóstico de neumotórax, los que tenían un origen traumático fueron excluidos, obteniéndose 3 casos; además de realizar una revisión actual de la bibliografía con relación al tema. Resultados. Un total de 6 pacientes tuvieron diagnóstico de neumotórax más COVID 19 , de los cuales dos pacientes se excluyeron porque la causa de su neumotórax se filio como traumática (accidentes de tránsito), 1 de los pacientes se descartó por no contar con datos suficientes del desenlace de su evolución clínica; del resto de los tres que se presentan en el artículo: 2 presentaron neumotórax espontáneo y en etapa tardía de la enfermedad, uno de los cuales fallece; 1 mostró neumotórax asociado a barotrauma por ventilación mecánica invasiva con un curso clínico favorable. Conclusión. El neumotórax a pesar de ser una complicación infrecuente en el espectro de la enfermedad por COVID 19, debe abordarse dentro del diagnóstico diferencial cuando un paciente presenta una descompensación respiratoria súbita; se requiere de mayores estudios para determinar si el neumotórax en estos pacientes conduce a 
current literature are based on a series of isolated cases.

Keywords: Pneumothorax, COVID 19, SARS CoV 2, Barotrauma, Pandemic. una mayor mortalidad, debido a que los reportes de la literatura actual están basados en serie de casos aislados.

Palabras clave: Neumotórax, COVID 19, SARS CoV 2, Barotrauma, Pandemia.

\section{Introducción.}

A finales del 2019 en Wuhan /China emergió un nuevo virus, que producía un síndrome de dificultad respiratoria aguda al que se llamó SARS CoV 2 , a pesar de la innumerables medidas para la contención de esta nueva enfermedad, el virus se esparció rápidamente alrededor de todo el mundo, siendo declarada por la Organización Mundial de la Salud (OMS) pandemia en marzo del 2020 (Elhakim et al., 2020).

Esta enfermedad ya ha tomado la vida de más de 1 millón de personas alrededor del mundo, si bien estamos conscientes de sus complicaciones más frecuentes, la mayoría desconoce aquellas que no son tan prevalentes, pero que tienen gran impacto en la morbilidad del paciente como es el neumotórax (Hernández Rivera et al., 2021).

El espectro clínico del COVID 19 puede ir desde los asintomáticos, casos leves, y en aproximadamente el $5 \%$ de los pacientes a desarrollar un compromiso severo con fallo multiorgánico (Alhakeem et al., 2020).

Si bien lo común es que los pacientes con COVID 19 que desarrollan insuficiencia respiratoria aguda lo hagan consecuentemente a la presencia del síndrome de dificultad respiratoria aguda propio de la enfermedad, no hay que dejar de lado otras complicaciones como la tromboembolia pulmonar y el neumotórax, que requieren un diagnóstico oportuno, porque su manejo terapéutico es diferente (Perez-Lopez \& Moreno-Madrigal, 2021).

La presencia de neumotórax es habitual en el paciente que se encuentra con ventilación mecánica invasiva (VMI), sin embargo ahora en el contexto de la actual pandemia por el SARS CoV 2 hay reportes de casos de neumotórax espontaneo como una complicación rara de la enfermedad, sin antecedente de barotrauma inducido por presión positiva en las vías respiratorias; en una serie de 99 pacientes publicada por Chen el neumotórax se presentó solo en el 1- 2\% de los pacientes con COVID 19. Estudios reportan una mediana de tiempo de 24.3 días desde la admisión hospitalaria para el desarrollo de esta complicación en el caso de ser espontaneo, y cuando se presenta por barotrauma en promedio se desarrolla al 4-5 día post-intubación (Quincho-Lopez et al., 2020) (Yuan et al., 2021)(Hernández Rivera et al., 2021).

El neumotórax es la acumulación de aire en el espacio pleural, lo que causa colapso pulmonar del lado comprometido, como se mencionó en el SARS CoV 2 este puede darse porque tal vez los pacientes tenían alguna condición pulmonar crónica previa predisponente, o por barotrauma provocado por VMI, o simplemente 
fisiopatológicamente se produce una lesión pulmonar directa difusa, que induce rotura alveolar y escape de aire al espacio pleural (Elhakim et al., 2020)(Hernández Rivera et al., 2021) (Redondo-Sendino et al., 2021).

Se clasifica en primario: sin enfermedad pulmonar subyacente; y secundario por ejemplo a: Enfermedad Pulmonar Obstructiva Crónica (EPOC), trauma, neoplasias, asma grave, neumonía necrotizante, etc.(Hernández Rivera et al., 2021). La clínica se caracteriza por disnea de aparición brusca, taquipnea y dolor torácico incluso en reposo pleurítico; dentro de la exploración física encontramos: abolición del murmullo vesicular, timpanismo a la percusión, disminución de la expansibilidad torácica del lado afectado; aquel paciente con COVID 19 que presente una descompensación brusca del cuadro respiratorio, o aquellos que están con VMI y se documenta desaturación súbita se pensará como alternativa diagnostica la posibilidad de neumotórax (Hernández Rivera et al., 2021) (Redondo-Sendino et al., 2021).

Para corroborar el diagnóstico se requiere de un método de imagen (radiografía de tórax, ecografía pulmonar, tomografía axial computarizada de tórax), la radiografía de tórax es normal en el $74 \%$ de los pacientes en la fase inicial del COVID 19, siendo que la bibliografía menciona que cuando el neumotórax se presenta regularmente lo hace en una fase tardía de la enfermedad es importante realizar una tomografía axial computarizada de tórax inicial y luego en controles subsecuentes; analizando estudios tomográficos de pacientes con COVID 19 que desarrollaron neumotórax un indicio común fue hallar bullas y neumatoceles. El tratamiento es en base a colocación de tubo torácico para su drenaje, alcanzándose la reexpansión pulmonar en la mayoría de los casos en un lapso de 48- 72 horas (Hernández Rivera et al., 2021) (Redondo-Sendino et al., 2021)(Sadiq et al., 2020).

Es importante ser proactivos en la búsqueda de esta posible complicación, debido a que la tasa de mortalidad se eleva sustancialmente en estos pacientes hasta en un 33\% (PerezLopez \& Moreno-Madrigal, 2021).

\section{Metodología.}

Se realizó búsqueda de pacientes con diagnóstico de neumotórax asociado a COVID 19, internados en el área de Aislamiento Respiratorio del Hospital Regional Ambato entre los periodos de febrero a julio del 2021, se excluyó dos paciente cuya causa de neumotórax fue traumática (accidente de tránsito), y un paciente cuya evolución no se pudo seguir en el tiempo, al final reportamos 3 casos en este artículo; la recolección de los datos se tomó de informes clínicos, respetando los aspectos de confidencialidad. Se revisa el perfil clínico, los estudios de imagen y el tratamiento recibido de los pacientes en cuestión. De manera conjunta se llevó a cabo una búsqueda sistemática de artículos de relevancia en PubMed, Medline, Google Scholar, Embase, Cochrane, usando los siguientes términos: Severe Acute Respiratory Syndrome, Síndrome Respiratorio Agudo Severo, coronavirus 2, COVID-19, SARS-CoV-2 y pneumothorax, neumotórax, complications, complicaciones. Se tomó en cuenta estudios desarrollados a partir del año 2019, que es cuando comenzó esta nueva enfermedad, en idioma español e inglés. 


\section{Descripción de los Casos Clínicos:}

\section{CASO 1}

Paciente femenina de 77 años con antecedente de hipertensión arterial y COVID-19 diagnosticado en diciembre del 2020, fue colocada tubo torácico por Neumotórax espontaneo el 26/02/21, paciente luego de 5 días se auto retira el tubo torácico y es referida a nuestro hospital, por cuadro de disnea. Ingresa con los siguientes signos vitales: tensión arterial (TA): 136/95 mm Hg , frecuencia cardiaca (FC): 86 por minuto, frecuencia respiratoria (FR): 24 por minuto, saturación de oxígeno (Sat O2): 93\% por cánula a 3 litros y con los siguientes exámenes de laboratorio: Leucocitos: 6840, hemoglobina: 11.4 , hematocrito: 36.2 , plaquetas:239.000, neutrófilos: 67.8 , linfocitos: 17.2, glucosa: 114.7 , urea: 13.7 , creatinina: 0.46 , ácido úrico: 2.7 , bilirrubina directa: 0.13, TGO: 42, TGP: 24, lactato deshidrogenasa: 189, sodio: 142, potasio: 3.28, cloro: 106, PCR-67.1, serología COVID 19 IGG e IGM positivas.

Se coloca tubo torácico por segunda ocasión en esta casa de salud el 03/03/2021, 48 horas después retiran tubo torácico, estudio de control con adecuada expansibilidad torácica, presenta diminución de necesidad de oxígeno, sin presentar signos de dificultad respiratoria, motivo por el cual se decide su alta con oxígeno suplementario a 1 litro por cánula nasal.

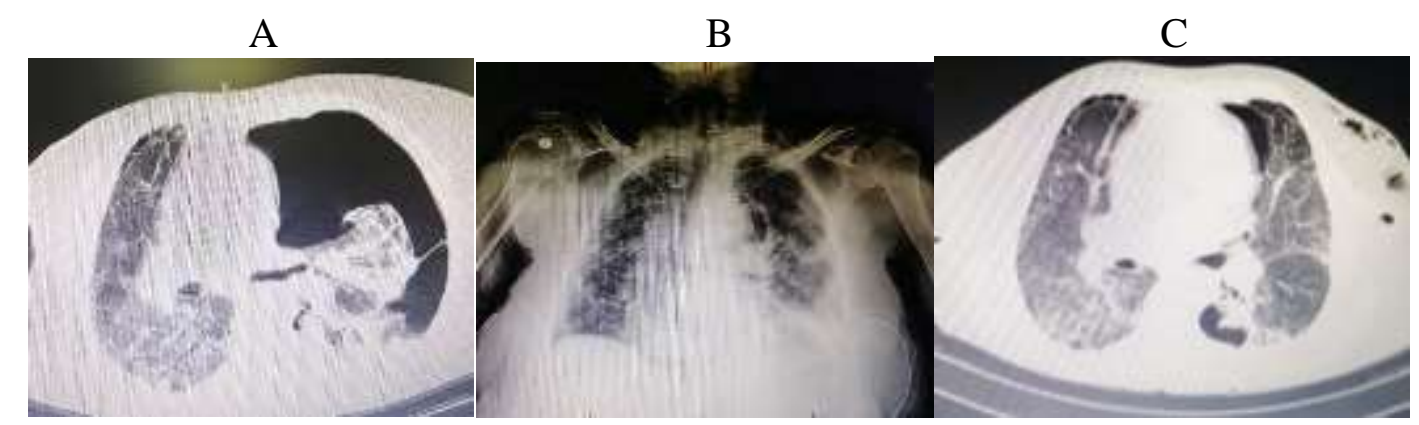

Figura 1, Servicio Imagen - Hospital Docente Regional Ambato. A: TAC de tórax inicial que demuestra gran neumotórax izquierdo. B: Rx de control post-colocación tubo torácico. C: Estudio tomográfico de seguimiento en el que se evidencia gran reabsorción del neumotórax.

\section{CASO 2}

Paciente masculino de 88 años con antecedente de hipertensión arterial, ingresa el 23/07/2021 por cuadro de 20 horas de disminución del estado de conciencia, sin causa aparente, acuden a médico particular quien coloca medicación intravenosa no especificada, sin mejoría por lo que es traído a emergencias en donde se evidencia desaturación, se realiza prueba rápida para COVID 19 cuantitativa positiva, al ingreso signos vitales: TA: 139/56 mm Hg. FC: 57, FR: 25, SAT O2: 90\% con FIO2 de 97\% con mascarilla de alto flujo, con los siguientes exámenes de laboratorio: leucocitos: 10450, hemoglobina: 12.7 , hematocrito: 36.6 , plaquetas 187.000, neutrófilos: 85.2 , linfocitos: 7.3 , glucosa 87 , urea 37 , creatinina 1.26 , sodio: 131 , potasio: 4.88 , cloro: 97 . En TAC de tórax se observa secuelas probablemente postcovid + neumotórax derecho, se 
interconsulta a cirugía para colocación de tubo torácico; que realiza la colocación de tubo torácico el cual se mantiene por 6 días. Nunca se filio causa orgánica para justificar estado de conciencia inicial y paciente no tuvo focalidad neurológica, fallece el 29/07/2021 debido a parada cardiorrespiratoria.

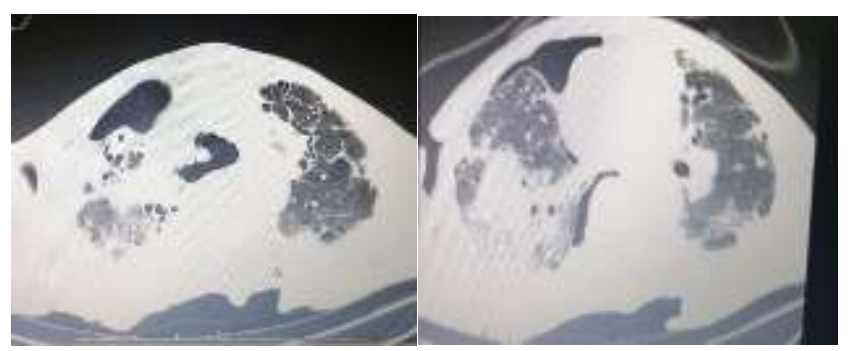

Figura 2, Servicio Imagen - Hospital Docente Regional Ambato. Imagen tomográfica con neumotórax derecho anterior y posteromedial derecho.

\section{CASO 3}

Paciente masculino de 27 años sin antecedentes de importancia ingresa el 09/07/2021 al servicio de aislamiento por dificultad respiratoria, con los siguientes signos vitales: TA: 140/75, FC: 110, FR: 45, SATO2: 84\% con mascarilla de alto flujo con FIO2 de 97\%, debido al cuadro clínico se decide su ingreso a unidad de cuidados intensivos en donde permanece del 10/07/2021 al 28/07/2021, intubado por 15 días, paciente mientras estuvo intubado presento neumotórax derecho que requirió colocación de tubo de tórax con su retiro el 26/7/21.

Recibe tratamiento antibiótico de amplio espectro, el 22/7/21 presenta descompensación hemodinámica más SIRS por lo que inician de forma empírica Colistin más Meropenem además en los cultivos se encuentra uno positivo de conducto auditivo (19/07/21) con Pseudomona fluorences sensible a Carbapenémicos; hemocultivos positivos por 2 (19/07/21) Pseudomona luteola con resistencia a Tigeciclina y el 26/07/21 presenta deposiciones liquidas con coprocultivo con Klebsiella pneumoniae multirresistente incluido Colistin.

Posterior a su estadía en unidad de cuidados intensivos, paciente es reingresado al servicio de aislamiento: 29/07/2021 en donde permanece con oxígeno suplementario a bajo flujo lográndose progresivamente su destete completo y cumpliendo curso completo de antibióticos, se da de alta el 07/08/2021.

A

B



Figura 3, Servicio Imagen - Hospital Docente Regional Ambato. A: Neumotórax derecho inducido por Barotrauma por VMI. B: TAC de tórax de control con resolución completa de neumotórax. 


\section{Discusión.}

El SARS CoV 2 es producido por un virus de ácido ribonucleico (ARN), su transmisión es a través de gotas expulsadas mediante estornudos o accesos de tos del paciente contaminado, también se ha mencionado los aerosoles como fuente de contagio y el contacto con superficies contaminadas como una forma menos frecuente; sus manifestaciones clínicas en la mayoría de pacientes son leves y lo más común es presentar: tos no productiva, malestar general, fiebre, disnea, nauseas, vómitos, diarreas, disminución o pérdida del olfato y el gusto. Un porcentaje menor de pacientes se complica y puede desarrollar un síndrome de dificultad respiratoria aguda grave, fallo multiorgánico, sepsis e incluso fallecer (Elhakim et al., 2020).

Por otro lado refiriéndonos al neumotórax, entre las circunstancias que pueden predisponer al desarrollo de uno espontaneo están: ser del género masculino, delgadez, talla alta, edad entre 10-30 años, tos excesiva, ejercicio extenuante; como etiologías del neumotórax secundario se nombran: trauma, infecciones, tabaquismo, déficit de alfa 1 antitripsina, enfermedades pulmonares crónicas tipo enfisema, enfermedades pulmonares intersticiales, enfermedades del tejido conectivo, tumores, etc.(Alhakeem et al., 2020) (Redondo-Sendino et al., 2021) (Perez-Lopez \& Moreno-Madrigal, 2021).

Varios procesos infecciosos de las vías respiratorias altas y bajas pueden ser una causa de neumotórax entre los cuales tenemos: neumonía micótica, estafilocócica, por Mycoplasma pneumoniae, neumonía organizada, pertussis, Pneumocistis carinni y tuberculosis en el paciente con Virus de Inmunodeficiencia Humana (VIH). Es raro que las infecciones virales sean causa de neumotórax y los virus asociados son: citomegalovirus e influenza principalmente (Elhakim et al., 2020) (Redondo-Sendino et al., 2021).

De los casos de pacientes presentados en el artículo, los dos primeros no tuvieron alguna patología previa identificada de manera que se catalogaron como primarios espontáneos por la infección por COVID 19, mientras el tercer caso presentó neumotórax secundario a barotrauma por VMI, además de tener varias infecciones graves bacterianas que también fueron condiciones propicias para el desarrollo del mismo.

La fisiopatología para el desarrollo de neumotórax en el entorno del COVID 19 se da por: las citocinas desencadenan un daño severo inflamatorio a las paredes alveolares y bronquiales, haciéndolas débiles; todo esto sumado a la congestión vascular, microtrombos y edema predisponen a la ruptura alveolar y de bullas preexistentes (Alhakeem et al., 2020). Otro mecanismo mencionado es la tos profusa (efecto Macklin) que aumenta la presión intrapulmonar lo que puede hacer que se rompan las bullas, que se forman por el daño alveolar y distorsión bronquial producida por la inflamación excesiva (Al-Shokri et al., 2020).

Se ha establecido además un daño isquémico inducido por COVID 19, ello predispone a la activación de fibroblastos conllevando a fibrosis pulmonar, sumado a las citocinas inflamatorias, causa que los alveolos se llenen de secreciones y así se provoque 
obstrucción con un efecto de válvula que es el sustrato para la formación de quistes; las disposición de estos últimos es de predominio periférico, lo que justifica que la ruptura de alguno de ellos lleve al desarrollo del neumotórax (Perez-Lopez \& Moreno-Madrigal, 2021).

Cerca de un tercera parte de los pacientes con COVID 19 hospitalizados desarrollan SDRA, el neumotórax como complicación es más frecuente en este último grupo (48.8\%), y esto se correlaciona con una mayor mortalidad; también es habitual que los pacientes que se encuentran con VMI presenten neumotórax inducido por barotrauma, es fundamental identificar prontamente estas circunstancias para evitar el deterioro hemodinámico y ventilatorio en el paciente (Dhanasopon et al., 2020). En una cohorte de 1099 pacientes en China el 15\% de ellos tuvo neumonía grave, de los cuales el 19\% ingreso a Unidad de Cuidados Intensivo (UTI) y aproximadamente la mitad requirió VMI o VNMI; un estudio realizado en New York determinó una incidencia de barotrauma asociados a VMI y COVID 19 en el 24\% de pacientes, y esta variable condicionó un mayor tiempo de hospitalización (Parra Gordo et al., 2021).

Hay que tener en cuenta además que bajo un mecanismo fisiopatológico similar también pueden haber otras complicaciones en el paciente con COVID 19 como el neumomediastino, enfisema subcutáneo, neumopericardio que pueden presentarse de manera aislada o en conjunto con el neumotórax (Hernández Rivera et al., 2021).

El tiempo que un paciente permanece intubado es un determinante esencial para el desarrollo de neumotórax, esta patología es más prevalente el los individuos que permanecen con VMI por más de 2 semanas (80\%) vs los que están con soporte ventilatorio menor a 1 semana (30\%) (Hernández Rivera et al., 2021).

Los neumotórax por barotrauma tienen una correlación elevada ante la presencia de los siguientes factores: duración de Síndrome Distrés Respiratorio Agudo (SDRA), presencia de EPOC, presiones inspiratorias pico mayores a 40-50 cms H2O, volúmenes corrientes elevados, presiones positivas al final de la espiración altas (Hernández Rivera et al., 2021).

Existe incluso bibliografía que indica el desarrollo de neumotórax en paciente con neumonía por SARS CoV 2 que se encontraban con cánula nasal de alto flujo, como terapia puente a VMI , subsecuente a barotrauma (Navarrete \& Mix, 2021).

Los hallazgos radiológicos más comunes a presentarse en la neumonía por COVID 19 son: opacidades en vidrio esmerilado, además de reticulación; su distribución es predominantemente periférica, bilateral y de disposición medio-basal; la presencia de consolidaciones constituye una fase tardía de la enfermedad (Elhakim et al., 2020) (Razek et al., 2021)(Sadiq et al., 2020).

La ecografía pulmonar al pie de la cama del paciente también es una herramienta útil, y que ha mostrado buena correlación con los hallazgos encontrados en la tomografía axial computarizada de tórax, y tiene la ventaja sobre ésta, en que se evitaría la movilización 
del paciente fuera del área de aislamiento, con ello disminuir el riesgo transmisibilidad por el traslado de los pacientes y se evitaría la desinfección posterior a la que deben someterse las salas de radiología; nos ayuda a establecer el grado de afectación pulmonar y las probables complicaciones como neumotórax y derrame pleural. La ecografía pleuropulmonar puede detectar neumotórax con una sensibilidad del $75 \%$ y especificidad del 98\% (Fraile Gutiérrez et al., 2020). Ecográficamente los hallazgos que indican presencia de neumotórax son ausencia de deslizamiento y presencia de líneas B (Hernández Rivera et al., 2021).

En un paciente con neumonía por COVID 19, cuando sufren una descompensación aguda se debe sospechar primero que la etiología sea la propia infección, tromboembolia pulmonar y no se puede dejar de lado otras complicaciones aunque menos prevalentes como el neumotórax (Alhakeem et al., 2020).

La limitación que existe para revisar minuciosamente a estos pacientes, por el riesgo de contagio, constituye un obstáculo, y muchas veces este diagnóstico puede pasar desapercibido (Al-Shokri et al., 2020).

Para el manejo del neumotórax asociado a COVID 19 , se prefiere la colocación de tubo torácico como terapia de primera línea sobre la aspiración con aguja, lo cual ha demostrado se eficaz y seguro, con notable mejoría de la oxigenación del paciente; el Colegio Americano de Cirugía torácica recomienda realizar drenaje cuando el neumotórax es mayor al 20\% (Yuan et al., 2021) (Hernández Rivera et al., 2021).

Hay que recordar que con la colocación de tubos de toracostomía existe alto riesgo de aerosolización, por lo que se deberá utilizar equipo de protección personal deacuerdo a la normativa del cada unidad hospitalaria, minimizar el personal en la habitación el momento del procedimiento, colocar un apósito oclusivo sobre el sitio de entrada del tubo torácico, estar a la vez atentos de infecciones nosocomiales que puedan tener una puerta de acceso a través de estos dispositivos y una vez retirado el drenaje todo el material debe ser desechado en doble bolsa de riesgo biológico (Hernández Rivera et al., 2021) (Dhanasopon et al., 2020).

En los casos de neumotórax persistentes, con fugas con una duración mayor a 3-5 días, cuando hay compromiso bilateral o presencia de hemo-neumotórax resulta útil la realización de toracostomía asistida por video (VATS), pleurectomía, mejorando considerablemente la mecánica ventilatoria de los pacientes. Como estrategias preventivas en el desarrollo del neumotórax inducido por barotrauma se utilizaran en VMI, volúmenes y presiones bajas (Hernández Rivera et al., 2021).

\section{Conclusiones.}

- El neumotórax es una rara complicación de la neumonía por SARS CoV 2, sin embargo su presencia puede tener un peor pronóstico en estos pacientes, se requieren estudios con mayor casuística para establecer una correlación significativa entre neumotórax / COVID 19 y mayor mortalidad. 
- Si existe empeoramiento en el curso clínico de un paciente con neumonía por SARS CoV 2, deben considerarse como alternativas en el diagnóstico diferencial complicaciones como la tromboembolia pulmonar, el neumotórax y neumomediastino, cuya identificación es esencial para el tratamiento oportuno de los mismos.

- Los cuadros más severos de neumonía por COVID 19, es decir aquellos que desarrollan SDRA, y además los que tienen un curso más prolongado de la enfermedad, son los más propensos a desarrollar neumotórax.

- Generalmente el neumotórax en el COVID 19 tiene una presentación tardía, por ende ante un paciente que aparentemente ya supero el proceso agudo, y nuevamente reingresa con dificultad respiratoria debemos descartar esta posibilidad diagnostica.

- Ante el riesgo de contagio latente en estos pacientes debemos ser más cautelosos en cumplir con todas las medidas de bioseguridad, debido a que la colocación de un tubo torácico es un procedimiento altamente generador de aerosoles.

\section{Referencias Bibliográficas.}

Al-Shokri, S. D., Ahmed, A. O. E., Saleh, A. O., AbouKamar, M., Ahmed, K., \& Mohamed, M. F. H. (2020). Case Report: COVID-19-related pneumothorax-case series highlighting a significant complication. American Journal of Tropical Medicine and Hygiene, 103(3), 1166-1169. https://doi.org/10.4269/ajtmh.20-0713

Alhakeem, A., Khan, M. M., Soub, H. Al, \& Yousaf, Z. (2020). Case Report: COVID19-Associated Bilateral Spontaneous Pneumothorax-A Literature Review. American Journal of Tropical Medicine and Hygiene, 103(3), 1162-1165. https://doi.org/10.4269/ajtmh.20-0680

Dhanasopon, A. P., Zurich, H., \& Preda, A. (2020). Chest Tube Drainage in the Age of COVID-19. Physician Assist Clin, 6(January), 261-265.

Elhakim, T. S., Abdul, H. S., Pelaez Romero, C., \& Rodriguez-Fuentes, Y. (2020). Spontaneous pneumomediastinum, pneumothorax and subcutaneous emphysema in COVID-19 pneumonia: A rare case and literature review. BMJ Case Reports, 13(12), 1-7. https://doi.org/10.1136/bcr-2020-239489

Fraile Gutiérrez, V., Ayuela Azcárate, J. M., Pérez-Torres, D., Zapata, L., Rodríguez Yakushev, A., \& Ochagavía, A. (2020). Ultrasound in the management of the critically ill patient with SARS-CoV-2 infection (COVID-19): narrative review TT - Ecografía en el manejo del paciente crítico con infección por SARS-CoV-2 (COVID-19): una revisión narrativa. Medicina Intensiva, 44(9), 551-565. https://pubmed.ncbi.nlm.nih.gov/32527471\%0Ahttps://www.ncbi.nlm.nih.gov/pmc /articles/PMC7198178/

Hernández Rivera, I., Pamanes Lozano, A., \& Bolívar Rodríguez, M. A. (2021). Neumotórax en pacientes COVID-19 . REVMEDUAS, 11(3), 258-268. 
Navarrete, F., \& Mix, A. (2021). Barotrauma en COVID-19: Asociación de neumotórax y uso de cánula nasal de alto flujo. ARS MEDICA, 46, 30-33.

Parra Gordo, M. L., Weiland, G. B., García, M. G., \& Choperena, G. A. (2021). Radiologic aspects of COVID-19 pneumonia: outcomes and thoracic complications. Radiologia, 63(1), 74-88. https://doi.org/10.1016/j.rx.2020.11.002

Perez-Lopez, K. P., \& Moreno-Madrigal, L. G. (2021). Spontaneous pneumothorax and pneumomediastinum in patients with pneumonia due to COVID-19. Medicina Interna de Mexico, 37(1), 152-156. https://doi.org/10.24245/mim.v37i1.4755

Quincho-Lopez, A., Quincho-Lopez, D. L., \& Hurtado-Medina, F. D. (2020). Case Report: Pneumothorax and Pneumomediastinum as Uncommon Complications of COVID-19 Pneumonia-Literature Review. American Journal of Tropical Medicine and Hygiene, 103(3), 1170-1176. https://doi.org/10.4269/ajtmh.20-0815

Razek, A., Fouda, N., Fahmy, D., Tanatawy, M. S., Sultan, A., Bilal, M., Zaki, M., AbdelAziz, M., \& Sobh, D. (2021). Computed tomography of the chest in patients with covid-19: What do radiologists want to know? Polish Journal of Radiology, 86(1), e122-e135. https://doi.org/10.5114/pjr.2021.104049

Redondo-Sendino, Gómez-Cuñarro, M., Jenkins-Sánchez, C. P., \& Redondo-Sendino, J. I. (2021). Pneumothorax associated with COVID-19. Semergen, 47(5), e35-e36. https://doi.org/10.1016/j.semerg.2020.11.004

Sadiq, Z., Rana, S., Mahfoud, Z., \& Raoof, A. (2020). Systematic review and metaanalysis of chest radiograph (CXR) findings in COVID-19. Clinical Imaging, 80(January), 229-238.

Yuan, X., Shanqing, L., \& Hongsheng, L. (2021). Clinical outcomes of pleural drainage on pneumothorax and hydrothorax in critically ill patients with COVID-19: A case series with literature review. Heart and Lung, 50(2), 213-219. https://doi.org/10.1016/j.hrtlng.2020.12.007

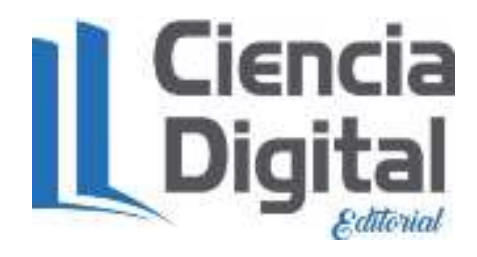




\section{PARA CITAR EL ARTÍCULO INDEXADO.}

Pinos Cedeño, M. J., Aguiar Flores, G. E., Layedra Ajila, M. E., \& Adriano Pérez, G. I. (2021). Neumotórax espontáneo y secundario a barotrauma en COVID 19, serie de casos y revisión de la literatura. Anatomía Digital, 4(4), 23-34. https://doi.org/10.33262/anatomiadigital.v4i4.1888

\section{\Ciencia}

El artículo que se publica es de exclusiva responsabilidad de los autores y no necesariamente reflejan el pensamiento de la Revista Anatomía Digital.

El artículo queda en propiedad de la revista y, por tanto, su publicación parcial y/o total en otro medio tiene que ser autorizado por el director de la Revista Anatomía Digital.
